Check for updates

Cite this: RSC Adv., 2017, 7, 29591

Received 18th April 2017

Accepted 25th May 2017

DOI: $10.1039 / \mathrm{c} 7 \mathrm{ra04366j}$

rsc.li/rsc-advances

\title{
Self-assembled nanoparticles based on poly(ethylene glycol)-oleanolic acid conjugates for co-delivery of anticancer drugs
}

\begin{abstract}
Yingsa Wang, ${ }^{\mathrm{a}}$ Yajie Tian, ${ }^{\mathrm{a}}$ Pengbo Zhu, ${ }^{\mathrm{a}}$ Yunyun $\mathrm{Ma},{ }^{\mathrm{a}}$ Jing He${ }^{\star \mathrm{ab}}$ and Jiandu Lei (D) *ab
Oleanolic acid (OA) has shown promising antitumor activity. However, it is difficult to formulate because of its poor water solubility and low bioavailability. In this study, self-assembled nanoparticles based on poly(ethylene glycol)-oleanolic acid conjugates (mPEG-OA) for co-delivery of anticancer drugs were developed to enhance the properties of OA. The mPEG-OA was synthesized by conjugating oleanolic acid (OA) to methoxy poly(ethylene glycol) carboxylic acid (mPEG-COOH). Then hydroxycamptothecin (HCPT), another anticancer drug was encapsulated into the mPEG-OA conjugates by the nanoprecipitation method. The MPEG-OA/HCPT nanoparticles (NPs) had an appropriate size, high drug loading of OA and HCPT, high stability, slow drug release and low cytotoxicity. In vivo studies revealed that the nanoparticles significantly improved the antitumor activity compared with free drugs. As no systemic toxicity was observed, the mPEG-OA NPs can serve as a promising drug delivery system for cancer therapy.
\end{abstract}

\section{Introduction}

Cancer is one of the most prevalent and deadly diseases worldwide. ${ }^{1}$ Existing chemotherapy is inadequate, with problems such as the increasing number of multidrug-resistant cancers and side effects of anticancer drugs. ${ }^{2}$ Drug delivery systems have been attracting considerable interest for a long time to improve the efficacy, reduce the toxicity and reduce

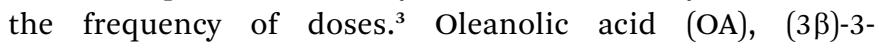
hydroxyolean-12-en-28-oic acid, is widely present in many Asian herbs. ${ }^{4}$ Although OA has been shown to have many significant biological actions including hepatoprotective, antiinflammatory, antioxidant and antitumor effects, the clinical application of OA has been limited by its poor aqueous solubility and extremely low dissolution rate in the gastrointestinal tract. ${ }^{5-7}$

Drug conjugation, as a prodrug strategy, provides the possibility to overcome these shortcomings, especially when combined with nanotechnology. ${ }^{8}$ Nanotechnology has evident merits in tumor therapy because of the improved accumulation of anticancer drugs in tumor tissues via the enhanced permeability and retention (EPR) effect. ${ }^{9-11}$ EPR is a passive targeting strategy for solid tumors mainly based on particle size. Angiogenic tumors tend to develop a permeable

${ }^{a}$ MOE Engineering Research Center of Forestry Biomass Materials and Bioenergy, Beijing Forestry University, Beijing, 100083, China. E-mail: hejing2008@sina.com; Fax: +86-10-62336349; Tel: +86-10-62336349

${ }^{b}$ Beijing Key Laboratory of Lignocellulosic Chemistry, Beijing Forestry University, Beijing, 100083, China vasculature and can selectively recruit circulating small particles. The hydrophobic drugs are usually modified by hydrophilic agents, such as polyethylene glycol (PEG), ${ }^{12}$ which serves as a versatile prodrug platform. Linear PEG is most widely used in drug modification due to its simple synthetic steps. ${ }^{13}$

Although PEG-drug conjugation has made significant improvements, such as improved water solubility, prolonged drug release and low toxicity, it suffers from other problems such as large dosage and drug resistance. Hence polytherapy is often used in the treatment of cancer. Self-assembled nanoparticles for drug co-delivery have gained considerable attention. ${ }^{14-16}$ The synergistic combination of two or more drugs can overcome undesirable toxicity and other side effects and consequently reduces the dosage of each agent. ${ }^{17}$

In this study, we designed a self-assembled nanoparticle based on poly(ethylene glycol)-oleanolic acid conjugates (mPEG-OA) as a drug delivery system. On the one hand, the anticancer drug OA interacted with methoxy poly(ethylene glycol) carboxylic acid (mPEG-COOH) via ester linkages and mPEG-OA was an amphiphilic structure which was able to selfassemble into micelles. On the other hand, hydroxycamptothecin (HCPT) was entrapped into mPEG-OA to achieve a combination therapy (Scheme 1). Finally, the biophysical properties of the MPEG-OA/HCPT nanoparticles (NPs), including particle size, loading efficiency, in vitro drug release kinetics, cellular uptake, in vivo antitumor activities and side effects were investigated. 


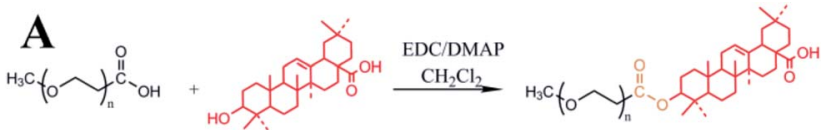

mPEG

OA

mPEG-OA
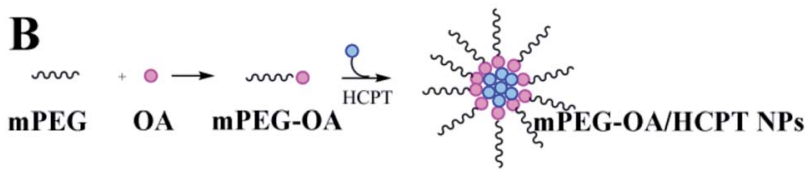

Scheme 1 Synthesis of MPEG-OA (A). Illustration of mPEG-OA codelivery of $\mathrm{HCPT}(\mathrm{B})$.

\section{Materials and methods}

\subsection{Materials}

Methoxy poly(ethylene glycol) carboxylic acid (mPEG-COOH, $M_{\mathrm{w}}=5000$ ) was purchased from Jenkem Technology Co., Ltd (Beijing, China). It is an FDA and EU approved food grade material. OA and HCPT were purchased from Chengdu Preferred Biotechnology Co., Ltd (Sichuan, China). 4-Dimethylaminopyridin (DMAP), dimethyl sulfoxide (DMSO) and 1-ethyl3-(3-dimethy-laminopropyl)-carbodiimide hydrogen chloride (EDC $\mathrm{HCl}$ ) were purchased from J\&K Chemical Reagent Co., Ltd (Beijing, China).

Penicillin and streptomycin, Gibco Dulbecco's PhosphateBuffered Saline (DPBS), and Gibco Dulbecco's Modified Eagle's Medium (DMEM) were purchased from Invitrogen. Fetal bovine serum (FBS) was purchased from HyClone. Cell-Counting Kit-8 (CCK-8) was supplied by Dojindo Laboratories. B16 murine melanoma (B16) cells were obtained from the Peking University Health Science Center (Beijing, China). The cells were cultured in the listed medium: DMEM with 10\% FBS and 1\% streptomycin-penicillin and maintained in an incubator supplied with a $5 \% \mathrm{CO}_{2}-95 \%$ air humidified atmosphere at $37{ }^{\circ} \mathrm{C}$.

Female $\mathrm{C} 57 \mathrm{BL} / 6$ mice, 6-7 weeks of age, were purchased from the National Institute for the Control of Pharmaceutical and Biological Products. All of the animal experiments were in accordance with the guidelines set by the National Institutes of Health (NIH Publication No. 85-23, revised 1985) and were approved by the Experimental Animal Ethics Committee, Beijing.

\subsection{Synthesis of MPEG-OA conjugate}

The mPEG-OA conjugate was synthesized via an esterification reaction by using DMAP as a catalyst and EDC as a coupling reagent in dichloromethane. Briefly, mPEG-COOH $(2.0 \mathrm{~g}, 0.4$ $\mathrm{mmol}$ ) and $\mathrm{OA}(1.5 \mathrm{~g}, 3.2 \mathrm{mmol})$ were dissolved in $20 \mathrm{~mL}$ of dichloromethane. The solution was cooled to $0{ }^{\circ} \mathrm{C}$ and EDC $(0.6 \mathrm{~g}, 1.8 \mathrm{mmol})$ and DMAP $(0.4 \mathrm{~g}, 3.2 \mathrm{mmol})$ were added to the mixture. The mixture was stirred at $0{ }^{\circ} \mathrm{C}$ for $1 \mathrm{~h}$ and at room temperature overnight. Then the solvent was evaporated under vacuum. The residue was dissolved in $10 \mathrm{~mL}$ of dichloromethane and the crude product was precipitated with ethyl ether $(100 \mathrm{~mL})$. After filtration the solids were washed with ethyl ether $(2 \times 100 \mathrm{~mL})$, and dried under vacuum to give mPEG-OA.

\subsection{Preparation of $\mathrm{mPEG}-\mathrm{OA} / \mathrm{HCPT}$ NPs}

Nanoparticles were prepared by a precipitation method according to previous reports. ${ }^{18}$ mPEG-OA conjugate $(10.0 \mathrm{mg})$ and free HCPT $(5.0 \mathrm{mg})$ dissolved in DMSO $(0.5 \mathrm{~mL})$ were added dropwise to aqueous media $(3.5 \mathrm{~mL})$, which was then vortexed for $10 \mathrm{~min}$ at room temperature. The resulting solutions were transferred to a MWCO 3500 cartridge, and dialyzed against normal saline $(100 \mathrm{~mL})$ for $3 \mathrm{~h}$, with two exchanges of dialysate.

\subsection{Determination of drug loading}

The drug loading efficiencies (DLE) of OA and HCPT were analyzed by a high performance liquid chromatography (HPLC) method with a UV detector set at $210 \mathrm{~nm}$ and $254 \mathrm{~nm}$, respectively. ${ }^{19} \mathrm{mPEG}-\mathrm{OA} / \mathrm{HCPT}$ NPs $(1.0 \mathrm{mg})$ were dissolved in acetonitrile $(1.0 \mathrm{~mL})$. The drug content in the mPEG-OA/HCPT NPs was determined using HPLC (OA: $210 \mathrm{~nm}, 95: 5$ mixture (v/v) of

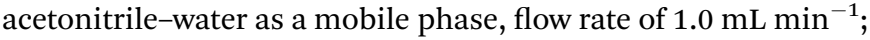
HCPT: $254 \mathrm{~nm}, 30: 70$ mixture (v/v) of acetonitrile-water as

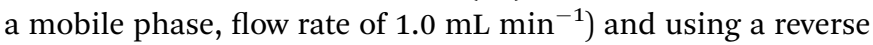
phase column (C18). The column temperature was $25{ }^{\circ} \mathrm{C}$. The DLE of OA and HCPT were calculated according to the following formula: DLE $(\mathrm{wt} \%)=$ (weight of loaded drug/weight of nanoparticles) $\times 100 \%$.

\subsection{In vitro drug release}

The release of OA and HCPT from mPEG-OA/HCPT NPs was analyzed by a dialysis method.$^{20}$ Firstly, mPEG-OA/HCPT NPs were diluted to $1.0 \mathrm{mg} \mathrm{mL}{ }^{-1}$, and then $5.0 \mathrm{~mL}$ of the solution was transferred to dialysis tubing (MWCO $3500 \mathrm{Da}$ ). The dialysis tubing was immersed into $100 \mathrm{~mL}$ of PBS (pH 7.4 or 5.5). Subsequently, the solution was shaken in a shaking water bath at $37^{\circ} \mathrm{C}$. At predetermined intervals, $2.0 \mathrm{~mL}$ of solution outside the dialysis bag was taken out and replaced by $2.0 \mathrm{~mL}$ of fresh PBS solution. The drug concentration in the medium was determined by HPLC. The release amount was then calculated. Each stability profile represents the average of three independent runs with the same sampling schedules. The standard deviation of each point is typically $2 \%$ or less. After 2 weeks, the solution inside the dialysis tubing was taken out and lyophilized, producing a white powder. ${ }^{1} \mathrm{H}-\mathrm{NMR}$ was used to demonstrate the hydrolysis.

\subsection{Characterization of mPEG-OA NPs and MPEG-OA/HC- PT NPs}

Proton nuclear magnetic resonance $\left({ }^{1} \mathbf{H}-\mathrm{NMR}\right)$. The ${ }^{1} \mathrm{H}-\mathrm{NMR}$ spectra were recorded on a Bruker Advance III 700M spectrometer with deuterated chloroform $\left(\mathrm{CDCl}_{3}\right)$ as solvent.

Dynamic light scattering (DLS). Mean particle size, polydispersity index and size distribution were determined using dynamic light scattering with a particle analyzer (Zetasizer Nano-ZS, Malvern Instruments Ltd, Malvern, UK). The samples were prepared by the methods mentioned before.

Transmission electron microscopy (TEM). The morphology of mPEG-OA NPs and mPEG-OA/HCPT NPs was verified by transmission electron microscopy (JEM-100CXa TEM). mPEG- 
OA and MPEG-OA/HCPT NPs were diluted $100 \times$ in deionized water, and a $2.0 \mu \mathrm{L}$ aliquot of solution was pipetted onto the surface of formvar coated copper TEM grids (TedPella, Redding, $\mathrm{CA}$ ) and allowed to air-dry. An acceleration voltage of $100 \mathrm{kV}$ was used.

\subsection{Solubility studies}

The solubility of mPEG-OA was analyzed. Briefly, excess amounts of MPEG-OA were added to screw-capped scintillation vials containing $10 \mathrm{~mL}$ of purified water. An aliquot of the sample $(5 \mathrm{~mL})$ was taken at $1 \mathrm{~h}$ intervals. The samples were analyzed directly by the HPLC method.

\subsection{In vitro cell cytotoxicity}

The cytotoxicity of free OA, free HCPT, mPEG-OA NPs and mPEG-OA/HCPT NPs was evaluated with a CCK-8 assay. ${ }^{21}$ B16 cells were used as a model of a tumor cell line. Briefly, the cell lines were seeded in 96-well plates (Corning, USA) at an initial density of $3 \times 10^{3}$ cells per well in $180 \mu \mathrm{L}$ of culture medium and incubated for $24 \mathrm{~h}$. Then, the cells were treated with various samples (OA, HCPT, mPEG-OA NPs and mPEG-OA/HCPT NPs) at $37{ }^{\circ} \mathrm{C}$ in a humidified incubator with $5 \% \mathrm{CO}_{2}$ for $48 \mathrm{~h}$ and $72 \mathrm{~h}$. The samples were prepared as follows. The samples of mPEG-OA NPs or mPEG-OA/HCPT NPs were dissolved in DMSO (Merck, Darmstadt, Germany) and then diluted in tissue culture medium. Free OA or HCPT of equivalent concentrations were added in parallel for comparison. The cells were treated with various concentrations of those drugs. $20 \mu \mathrm{L}$ of CCK- 8 solution was added to each well and incubated for $1 \mathrm{~h}$ at $37{ }^{\circ} \mathrm{C}$. The percentage cell viability can be calculated by measuring the absence of absorption at $450 \mathrm{~nm}$. $\mathrm{IC}_{50}$ was calculated as polymer concentrations which inhibited the growth of $50 \%$ of the $\mathrm{B} 16$ cells. $\mathrm{IC}_{50}$ was calculated using the Boltzmann sigmoidal function from Origin 8.6 (OriginLab, Northampton, USA). Data are representative of three independent experiments.

We evaluate the synergistic effects between OA and HCPT in the mPEG-OA/HCPT NPs by applying the combination index $(\mathrm{CI})=\mathrm{OA}_{\text {combined }} / \mathrm{OA}_{\text {single }}+\mathrm{HCPT}_{\text {combined }} / \mathrm{HCPT}_{\text {single }}$, whereby $\mathrm{OA}_{\text {combined }}$ and $\mathrm{HCPT}_{\text {combined }}$ represent the $\mathrm{IC}_{50}$ of drugs used in the combination treatment, and $\mathrm{OA}_{\text {single }}$ and $\mathrm{HCPT}_{\text {single }}$ represent single drug $\mathrm{IC}_{50}$. An index lower than 1 denotes drug synergism while one larger than 1 shows an antagonistic effect. ${ }^{22}$

\subsection{Cellular uptake study}

The cellular uptake of HCPT from the MPEG-OA/HCPT NPs was evaluated by flow cytometry (CaAn ADP9, Beckman Coulter, America). B16 cells were seeded in a 12-well tissue culture plate ( $2 \mathrm{~mL}$ medium) at a density of $2 \times 10^{5}$ cells per well. After being cultured for $24 \mathrm{~h}$, the original medium was replaced by HCPT and mPEG-OA/HCPT NPs $\left(\mathrm{IC}_{50}\right)$ and incubated for $4 \mathrm{~h}$ at $37^{\circ} \mathrm{C}$. After the incubation, the cells were washed with PBS (pH 7.4) twice and treated with trypsin $(0.5 \mathrm{~mL})$ for $2 \mathrm{~min}$. Then the cells were resuspended in PBS $(2 \mathrm{~mL})$ and subjected to the flow cytometry analysis.

\subsection{In vivo antitumor efficiency}

A tumor xenograft model was established in the right axillary flank region of female C57BL/6 mice (6-7 weeks) after injection of $3 \times 10^{6}$ B16 cells. Treatments were initiated when tumors reached an average volume of 100 to $150 \mathrm{~mm}^{3}$, and this day was designated as day 0 . On day 0 , these mice were randomly divided into 6 groups $(n=6)$ and administered intravenous injection with PBS (control), free OA $(10 \mathrm{mg}$ $\mathrm{kg}^{-1}$ ), free HCPT (10 $\left.\mathrm{mg} \mathrm{kg}^{-1}\right)$, OA/HCPT (OA: $4 \mathrm{mg} \mathrm{kg}^{-1}$, HCPT: $\left.10 \mathrm{mg} \mathrm{kg}^{-1}\right)$, mPEG-OA NPs $\left(10 \mathrm{mg} \mathrm{kg}^{-1}\right.$, OA equivalent), or mPEG-OA/HCPT NPs (10 mg kg ${ }^{-1}$, HCPT equivalent) every other day until day 8 . All groups were intravenously injected via the tail vein. In the observation phase, the tumor sizes and body weights were monitored every other day. The tumor volume was calculated using the formula: $\left(L \times W^{2}\right)$ / 2 , where $L$ is the longest and $W$ is the shortest tumor diameter (millimeter). For efficacy studies, the percentage of tumor growth inhibition (\% TGI) was calculated using the following formula: $[(C-T) / C] \times 100 \%$, where $C$ is the mean tumor volume of the control group at a specified time and $T$ is the mean tumor volume of the treatment group at the same time. At the end of the experiment ( $>6$ weeks) or when the implanted tumor volume reached $5000 \mathrm{~mm}^{3}$, the mice were sacrificed for humane reasons. To further evaluate the hematological toxicity of the nanoparticles, $200 \mu \mathrm{L}$ of blood was sampled from each mouse after final administration to test the white blood cell number (WBC) using a hematology analyzer (MEK-7222K, Nihon Kohden Celltac E).

\subsection{Side effects}

Toxic side-effects of current chemotherapeutical drugs often lead to a severe reduction in the quality of life, so the detection of allergic reactions is very important. Type I hypersensitivity is the most common type of hypersensitivity reaction and IgE antibodies play an important role in mediating type I hypersensitivity responses. ${ }^{23,24}$ Thus we selected IgE levels as the parameter for rapid evaluation of type I hypersensitivity reactions. Six groups of tumor-bearing mice (26-28 g, $n=6)$ were used in allergy testing studies (control, OA, HCPT, OA/ HCPT, mPEG-OA NPs, mPEG-OA/HCPT NPs). The samples were administrated via tail intravenous injection every other day at the OA and HCPT doses of $10 \mathrm{mg} \mathrm{kg}^{-1}$ body weight. After administration with different samples for 10 days, the orbit blood of mice in different groups was collected and centrifuged. Serum samples were analyzed according to the procedure of mouse IgE ELISA.

\subsection{Statistical analysis}

All experiments in this study were performed three times, and the data were expressed as the means \pm standard deviation (SD). Analysis of variance (ANOVA) was utilized to determine statistical significance between different groups. In all the analyses, $p<0.05$ was considered to be of statistical significance. 


\section{Results and discussion}

\subsection{Synthesis of mPEG-OA conjugate}

A conjugate made of hydrophilic $\mathrm{MPEG}$ and hydrophobic drug OA was successfully synthesized. Fig. 1 shows the ${ }^{1} \mathrm{H}-\mathrm{NMR}$ spectra of mPEG-OA and OA. In Fig. 1A, typical peaks at $5.3 \mathrm{ppm}$ (peak a) and 0.7-2.0 ppm were assigned to the protons of OA. After modification of OA, Fig. 1B, additional chemical signals at 2.8-3.9 ppm were detected and ascribed to the protons of $\mathrm{mPEG}$, indicating a chemical linkage between OA and MPEG. Due to the formation of ester bonds, the proton peak $\delta 3.2(1 \mathrm{H}, \mathrm{t})$ of OA moved to $\delta 4.2(1 \mathrm{H}, \mathrm{t})$. The signal of OA was significantly weakened and that of MPEG was detected, further indicating the successful synthesis of mPEG-OA.

\subsection{Characterization of the nanoparticles}

Nanoparticles were prepared by the nanoprecipitation method according to our previous report. ${ }^{18}$ The mPEG-OA conjugate and HCPT were dissolved in $0.5 \mathrm{~mL}$ of DMSO solution. Then the mixture was added dropwise into aqueous media. The loading efficiency on nanoparticles was calculated and is shown in Table 1. The mPEG-OA/HCPT NPs contained $6.18 \pm 1.20 \mathrm{wt} \%$ $\mathrm{OA}$ and $14.12 \pm 0.73 \mathrm{wt} \% \mathrm{HCPT}$, suggesting the successful loading of HCPT into the mPEG-OA NPs.

The desired diameters of the self-assembled nanoparticles were between 20 and $200 \mathrm{~nm}^{25}$ The diameters of the nanoparticles were measured by dynamic light scattering (DLS). Fig. 2 presents the size distributions of the mPEG-OA NPs and mPEG-OA/HCPT NPs. The surface zeta potential is a key factor
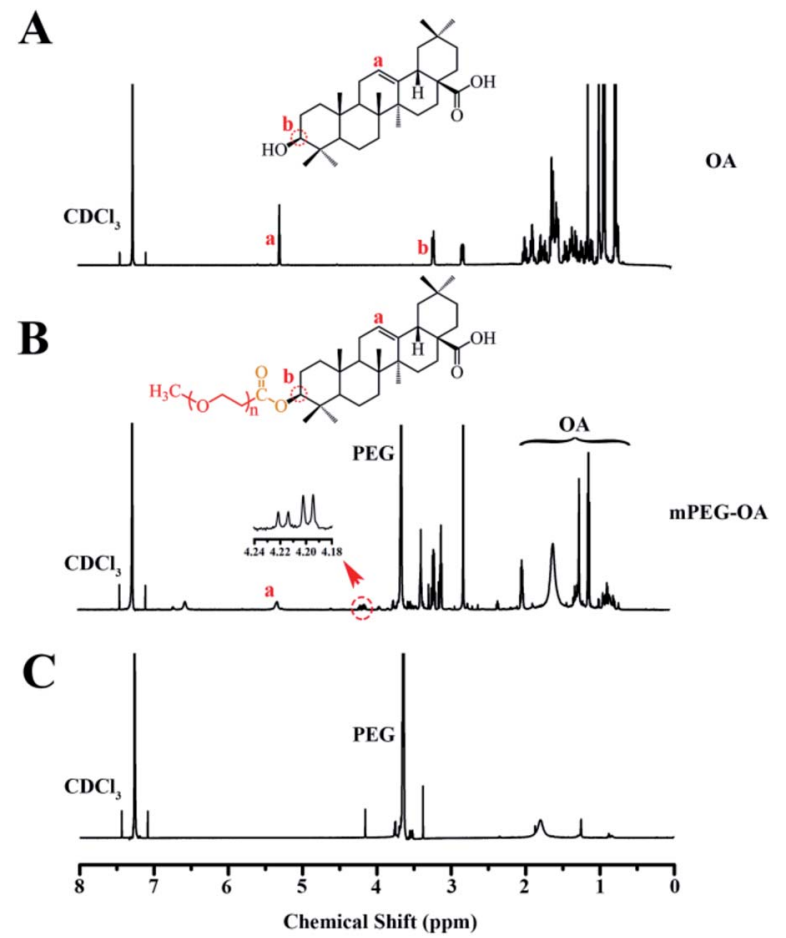

Fig. $1{ }^{1} \mathrm{H}$-NMR spectra of $\mathrm{OA}$ in $\mathrm{CDCl}_{3}(\mathrm{~A}), \mathrm{mPEG}-\mathrm{OA}$ in $\mathrm{CDCl}_{3}(\mathrm{~B})$. ${ }^{1} \mathrm{H}-\mathrm{NMR}$ spectra of the hydrolysis result of the $\mathrm{mPEG}-\mathrm{OA}(\mathrm{C})$. for better blood compatibility and prolonged circulation time of the nanoparticles for reduced clearance by the reticuloendothelial system (RES). ${ }^{26}$ The zeta potential of the nanoparticles was determined by a Nano-ZS Zeta Sizer. The size and the zeta potential of the nanoparticles are summarized in Table 1. It was observed that the average particle size of the MPEG-OA/HCPT NPs was larger than that of mPEG-OA NPs, which was attributed to the loaded drug. The final mPEG-OA/HCPT NPs had a diameter of $164.18 \pm 40.12 \mathrm{~nm}$ and a negative surface charge of $-5.13 \pm 0.28 \mathrm{mV}$ with a low polydispersity index. The negatively charged surface might be due to the carboxylate groups that resided on the outer surface of the nanoparticles after selfassembly in an aqueous solution. The carboxylate groups dominated the surface charge of the self-assembled nanoparticles.

\subsection{Solubility studies}

Solubility studies were carried out to explore the solubility of OA and $\mathrm{mPEG}-\mathrm{OA}$ in purified water. The results indicated that PEGylation significantly increased the solubility of OA. The solubility of OA was about $0.018 \mathrm{mg} \mathrm{mL}^{-1}$. The $\mathrm{mPEG}-\mathrm{OA}$ had $12.30 \mathrm{mg} \mathrm{mL}{ }^{-1}$ solubility, which was equivalent to $1.03 \mathrm{mg}$ $\mathrm{mL}^{-1}$ of OA (Table 1). The increased solubility makes it possible to systemically evaluate the therapeutic efficacy of OA in vivo.

\subsection{In vitro drug release}

The release profiles of the nanoparticles at two different $\mathrm{pH}$ were studied in PBS (pH 5.5 and 7.4) at $37^{\circ} \mathrm{C}$. The nanoparticles were dialyzed against PBS through a membrane with a cutoff size of $3500 \mathrm{Da}$. This particular size was selected as the membrane pores were large enough to allow the diffusion of free drug but not nanoparticles. The results indicated that the mPEG-OA NPs and the MPEG-OA/HCPT NPs slowly hydrolyzed and released OA in PBS ( $\mathrm{pH} 5.5$ and 7.4) without burst release (Fig. 3A and C). As shown in Fig. 1C, the peaks at $\delta 3.3-4.2$ were assigned to the methylene protons of the mPEG. And the OA typical peaks at 5.3 $\mathrm{ppm}$ and $0.7-2.0 \mathrm{ppm}$ disappeared from the original position. The results indicated that the OA were successfully released from the mPEG-OA.

As for HCPT, there was an initial burst followed by a sustained drug release (Fig. 3B). The slight initial burst release of HCPT indicated that HCPT was dispersed on and near the surface of the mPEG. It might also be attributed to balancing the equilibrium between the inside and outside release settings.

The $\mathrm{pH}$ of tumor tissue is much lower than that of normal tissue. ${ }^{27}$ Thus, the drug release profile of the $\mathrm{mPEG}-\mathrm{OA} / \mathrm{HCPT}$ NPs was also investigated at $\mathrm{pH}$ 5.5. It was clearly observed that the drug released from the MPEG-OA/HCPT NPs was higher at $\mathrm{pH} 5.5$ than at $\mathrm{pH} 7.4$ over a $150 \mathrm{~h}$ period. The result was due to the re-protonation of the hydroxyl group of OA. As the external $\mathrm{pH}$ decreased, the nanoparticles quickly hydrolyzed and released the OA and HCPT which could increase the toxicity to the tumor. Therefore, these findings enable the development of a new type of nanoparticle for drug delivery applications.

As shown in Fig. 3D, the average particle size of the nanoparticles hardly changed during the investigation period. The 
Table 1 Drug loading efficiency, size, zeta potential and solubility of the nanoparticles

\begin{tabular}{llllll}
\hline Compound & $\operatorname{DLE}_{\mathrm{OA}}(\mathrm{wt} \%)$ & $\operatorname{DLE}_{\mathrm{HCPT}}(\mathrm{wt} \%)$ & Size $(\mathrm{nm})$ & Zeta potential $(\mathrm{mV})^{\text {Solubility }\left(\mathrm{mg} \mathrm{mL}^{-1}\right)}$ \\
\hline OA & - & - & - & - & 0.0018 \\
mPEG-OA NPs & $6.20 \pm 0.82$ & - & $105.71 \pm 26.89$ & $-4.64 \pm 0.21$ & $12.30\left(1.03^{a}\right)$ \\
mPEG-OA/HCPT NPs & $6.18 \pm 1.20$ & $14.12 \pm 0.73$ & $164.18 \pm 40.12$ & $-5.13 \pm 0.28$ & -
\end{tabular}

${ }^{a}$ Equivalent to free OA.
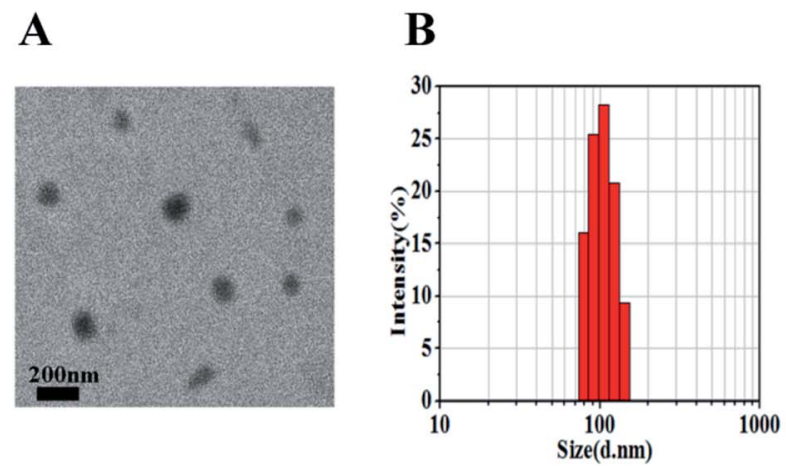

C

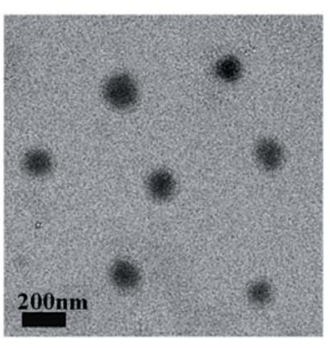

\section{D}

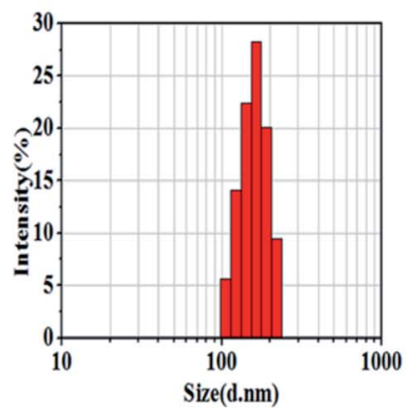

Fig. 2 mPEG-OA NPs (A and B) and mPEG-OA/HCPT NPs (C and D) observed by TEM (scale bar $=200 \mathrm{~nm})$ and DLS.

$\mathbf{A}$

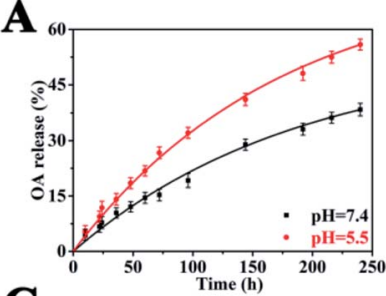

$\mathbf{C}$

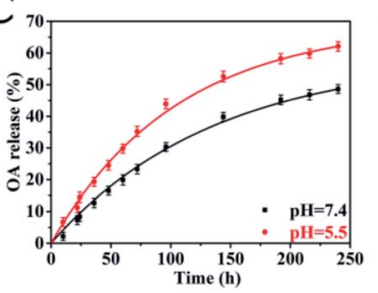

B

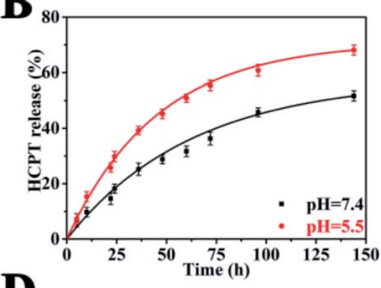

D

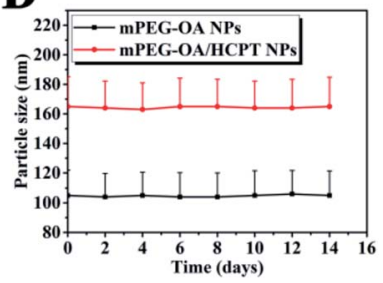

Fig. 3 Drug release curves in $\mathrm{PBS}$ at $37^{\circ} \mathrm{C}$ from the $\mathrm{mPEG}-\mathrm{OA} / \mathrm{HCPT}$ NPs (A: OA and B: HCPT) and the mPEG-OA NPs (C: OA). The size distribution of nanoparticles after 14 days of storage at $4{ }^{\circ} \mathrm{C}(\mathrm{D})$.

results indicated that the MPEG-OA NPs and the MPEG-OA/ HCPT NPs displayed a good redispersion stability.

\subsection{In vitro cell cytotoxicity}

The cell cytotoxicity was measured by cancer cell line viability. Free OA, free HCPT, mPEG-OA NPs and mPEG-OA/HCPT NPs were tested for their cytotoxic effects on B16 cells by CCK-8 assay. The cytotoxic effect of NPs was concentrationdependent. As shown in Fig. 4 and Table 2, $\mathrm{IC}_{50}$ was evaluated by survival curves. The results demonstrated that the $\mathrm{IC}_{50}$ values of the samples were ranked as OA > HCPT > mPEG-OA NPs > mPEG-OA/HCPT NPs. mPEG-OA/HCPT NPs provided more

efficient delivery of the drug compared to the others. Nanoparticles had a higher efficacy than the free drug because of the continuous exposure of the drug into the cells. Entrapment of HCPT into mPEG-OA NPs reduced the $\mathrm{IC}_{50}$ comparable of mPEG-OA NPs, which confirmed the synergy between OA and HCPT. The mPEG-OA/HCPT NPs (6.18 wt\% OA, 14.12 wt $\%$ HCPT) $\mathrm{IC}_{50}$ value of B16 cells was $1.37 \mu \mathrm{g} \mathrm{mL} \mathrm{m}^{-1}$ around 18 -fold lower than that of OA. The CI value of OA and HCPT in the mPEG-OA/HCPT NPs was 0.14, which is smaller than 1 . This result suggested that the MPEG-OA/HCPT NPs achieve a synergistic effect by co-delivery of two different anticancer drugs OA and HCPT.

\subsection{Cellular uptake study}

To investigate the cellular uptake, B16 cells were incubated with free HCPT and MPEG-OA/HCPT NPs for $4 \mathrm{~h}$. It was found that the HCPT fluorescence intensity in B16 cells treated with the mPEG-OA/HCPT NPs was stronger than that treated with free HCPT (Fig. 5A and B). The results indicated that the MPEG-OA/ HCPTNPs were efficiently endocytosed by B16 cells.

\subsection{In vivo antitumor efficiency}

On the basis of the above results, the antitumor effect of mPEGOA/HCPT NPs was explored in a mouse tumor model. The in vivo antitumor efficiency of the drug delivery nanoparticles was compared with free OA, free HCPT and OA/HCPT on a multipledose schedule in xenograft models of B16 murine melanoma. Tumor-bearing mice treated with the nanoparticles showed a prominent survival advantage compared with the control treated mice (Fig. 6B). The groups treated with free OA, free HCPT, OA/HCPT, mPEG-OA NPs and mPEG-OA/HCPT NPs 


- Control $"$ OA $\bullet$ HCPT $\vee$ mPEG-OA NPs $\$ mPEG-OA/HCPT NPs

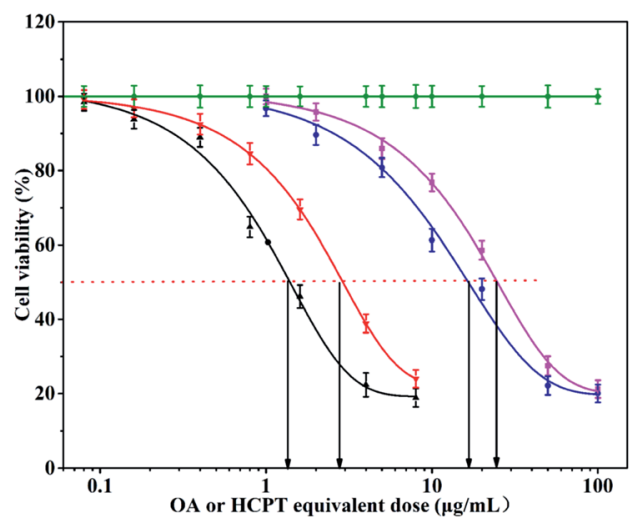

Fig. 4 Cellular cytotoxicity of control, OA, HCPT, mPEG-OA NPs and mPEG-OA/HCPT NPs in B16 cells. CCK-8 assay of OA, HCPT and nanoparticles with different concentrations (equivalent to native $O A$ and HCPT) in B16 cell lines ( $n=3$, error bars represent standard deviation)

Table 2 In vitro cytotoxicity analysis $\left(\mathrm{IC}_{50}, \mu \mathrm{g} \mathrm{mL}{ }^{-1}\right)$

\begin{tabular}{lc}
\hline Compound & $\begin{array}{l}\mathrm{B} 16 \\
(\mu \mathrm{g} \mathrm{mL})\end{array}$ \\
\hline OA & 25.04 \\
HCPT & 16.78 \\
mPEG-OA NPs & 2.82 \\
mPEG-OA/HCPT NPs & 1.37 \\
\hline
\end{tabular}

showed varied levels of antitumor effects and they were in order mPEG-OA/HCPT NPs $>$ mPEG-OA NPs $>$ OA/HCPT $>$ HCPT $>$ OA (Fig. 6C). mPEG-OA/HCPT NPs resulted in $82.7 \%$ TGI (day 20) and $50 \%$ survival of animals (day 30 ). mPEG-OA NPs resulted in $73.4 \%$ TGI (day 20 ) and $33.3 \%$ survival of animals (day 30 ). Free OA resulted in $49.1 \%$ TGI (day 20) and $0 \%$ survival of animals (day 30), free HCPT resulted in $46.7 \%$ TGI (day 20) and $16.7 \%$ survival of animals (day 30 ) and OA/HCPT resulted in $58.2 \%$ TGI (day 20) and $16.7 \%$ survival of animals (day 30) (Fig. 6A and B and Table 3).

The systemic toxicity was evaluated by monitoring the body weight. After drug administration every other day, the body weight of free OA, free HCPT, OA/HCPT, mPEG-OA NPs and MPEG-OA/HCPT NPs exhibited no statistically significant changes compared to the PBS control (Fig. 6D). The results indicated that no signs of systemic toxicity were observed. These studies demonstrated that mPEG-OA/ HCPT NPs have obviously mitigated the toxicities of the drug treatment while maintaining significant therapeutic efficacy.

\subsection{Evaluation of the side effects}

Many drug formulations, such as paclitaxel and docetaxel are always associated with serious side effects, including hematological toxicity and hypersensitivity reactions (usually type I hypersensitivity). The IgE antibodies play an important role in mediating type I hypersensitivity responses. Herein, we selected IgE levels as the parameter for rapid evaluation of type I hypersensitivity reactions. As shown in Fig. 6E, the blood IgE levels of mice in different groups (PBS, OA, HCPT, OA/HCPT, mPEG-OA NPs, mPEG-OA/HCPT NPs) were evaluated. Mice treated with OA, HCPT and OA/HCPT showed higher IgE levels than the PBS group, which might be due to the poor water solubility. The IgE levels of mPEG-OA NPs and mPEG-OA/ HCPT NPs were observed to be without significant change. This explored the idea that the use of these nanoparticles could distinctly reduce the risk of hypersensitivity reactions. The blood of mice was also collected to test the WBC count after treatment with different OA formulations. The WBC count is often used as an indicator of hematological toxicity. The results demonstrated that the WBC count of mice treated with OA displayed a small decrease over the normal group (Fig. 6F). No obvious decrease in the WBC number of the mice treated with MPEG-OA NPs and MPEG-OA/HCPT NPs was observed, indicating that the nanoparticles could avoid severe hematotoxicity.
A

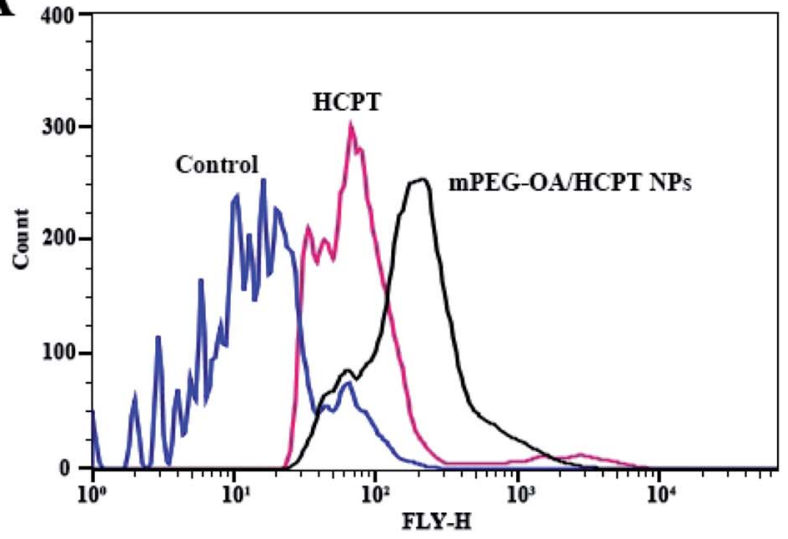

B

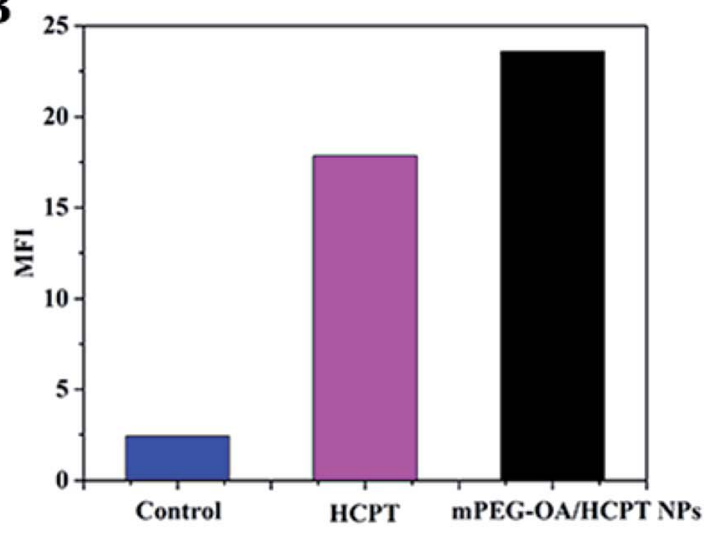

Fig. 5 Flow cytometry analysis (A) and MFI of B16 cells incubated with free HCPT and the mPEG-OA/HCPT NPs at $37{ }^{\circ} \mathrm{C}$ for $4 \mathrm{~h}$ (B). The untreated cells are used as a control. 
A

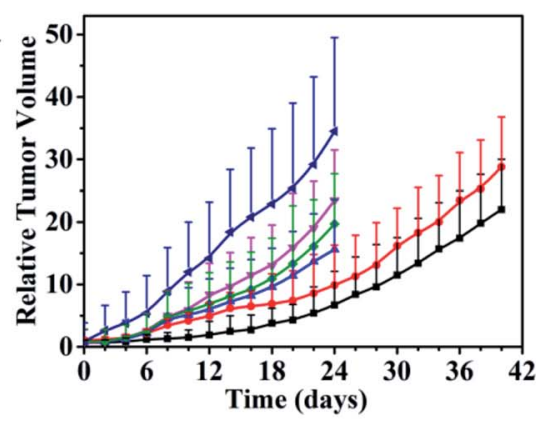

B

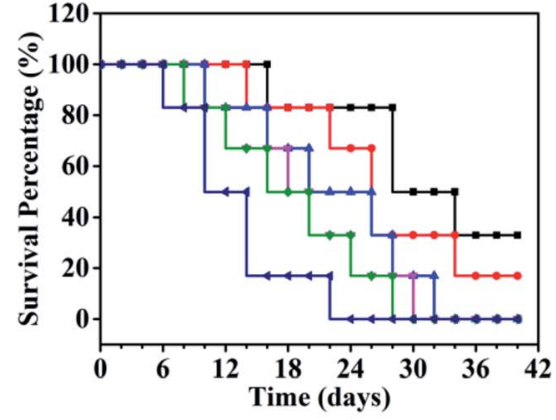

$\mathbf{C}$
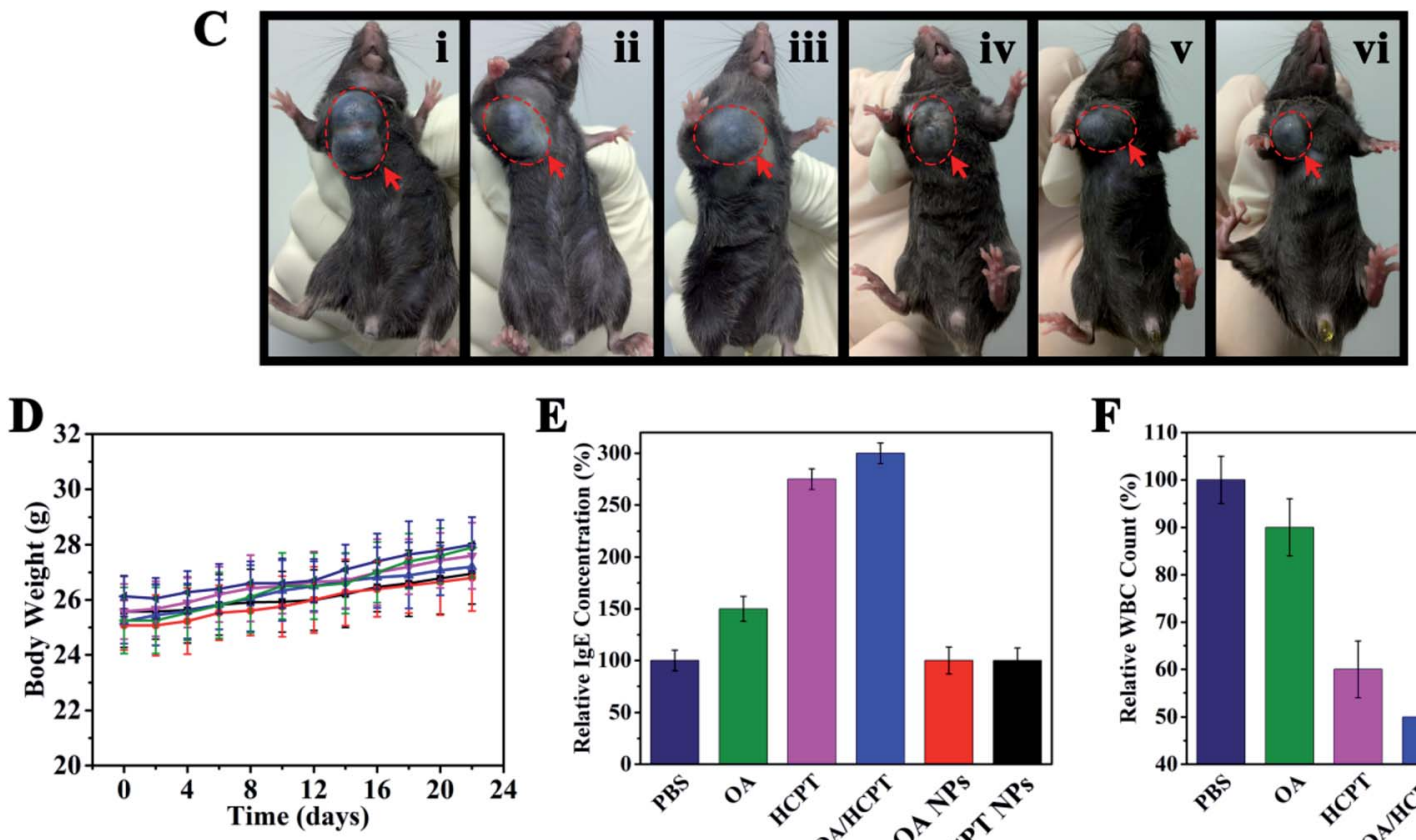

$\mathbf{E}$

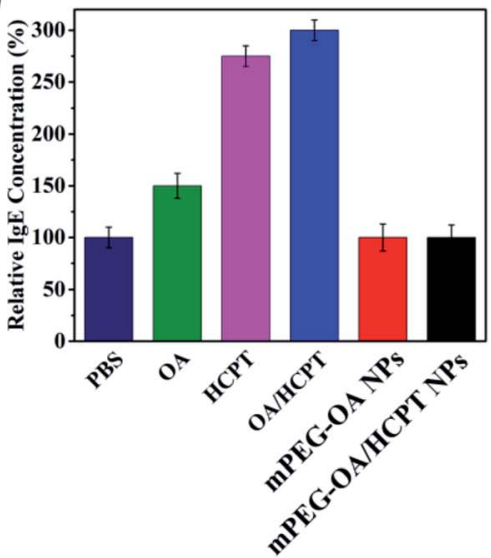

$\mathbf{F}$

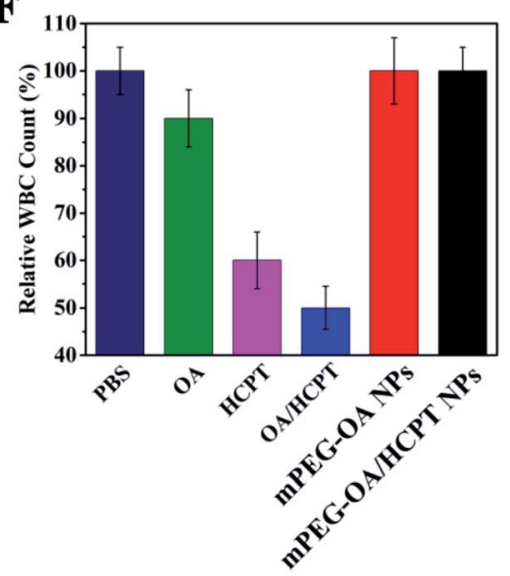

Fig. 6 Antitumor efficacy of OA, HCPT, OA/HCPT, mPEG-OA NPs, mPEG-OA/HCPT NPs in B16-bearing mice model. Tumor volumes of mice with different drug formulations; dashed line: stop chemotherapy (A). Survival percentage of different treatments (B). Tumor photograph from each treatment group excised on day 20 ((i): control, (ii): OA, (iii): HCPT, (iv): OA/HCPT, (v): mPEG-OA NPs, (vi): mPEG-OA/HCPT NPs) (C). Body weight variation of mice with different treatments (D). IgE levels of mice treated with different drug formulations for 30 min (E). WBC change during different administrations in normal mice. Blood samples were collected from mice on day 2 after the last treatment. Data are shown as means $\pm \mathrm{SD} ; n=6(\mathrm{~F})$.

Table 3 B16 xenograft model (q2d $\times 5$ ): efficacy comparison

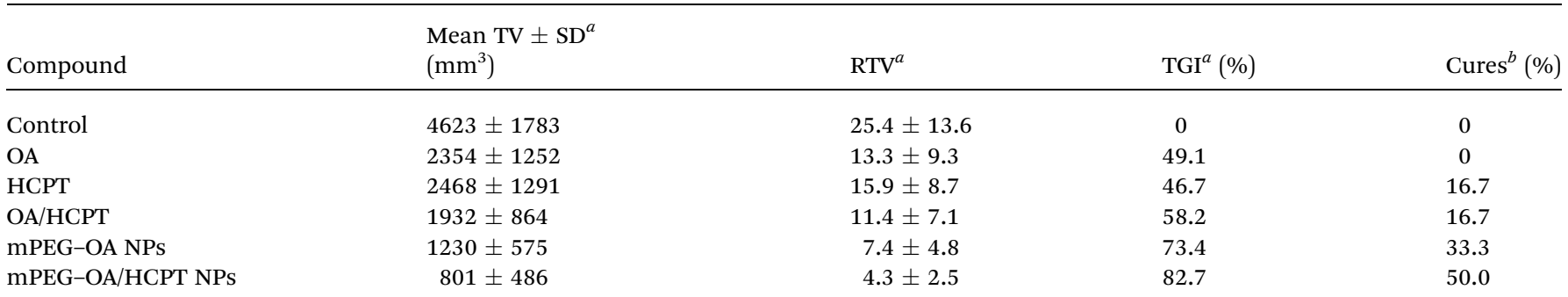

${ }^{a}$ Mean tumor volume (TV), RTV, and \% TGI data were taken at day 20 (by day 20, a significant percentage of control animals were euthanized due to excess tumor burden). ${ }^{b} \%$ cures were taken at day 30 . 


\section{Conclusions}

In our study, mPEG-OA nanoparticles with a hydrophobic core of OA and a hydrophilic PEG shell have been developed and successfully synthesized which can also encapsulate another anticancer drug HCPT to achieve a synergistic effect. By evaluating the anticancer effects of the nanoparticles, mPEG-OA/ HCPT NPs have shown significantly enhanced anticancer efficacy compared with free drug formulations. These nanoparticles for co-delivery of anticancer drugs also displayed a series of desirable properties, such as good water solubility, appropriate size and low side effects. It is believed that our study is a helpful and meaningful guide for future research.

\section{Acknowledgements}

This work was supported by the National Natural Science Foundation of China (No. 21576029), and the China State Forestry Administration 948 Project (No. 2014-4-35).

\section{Notes and references}

1 F. Bray, A. Jemal, N. Grey, J. Ferlay and D. Forman, Lancet Oncol., 2012, 13, 790-801.

2 T. Minko, L. Rodriguez-Rodriguez and V. Pozharov, Adv. Drug Delivery Rev., 2013, 65, 1880-1895.

3 S. Mura, J. Nicolas and P. Couvreur, Nat. Mater., 2013, 12, 991-1003.

4 J. Hao, J. Liu, X. A. Wen and H. B. Sun, Bioorg. Med. Chem. Lett., 2013, 23, 2074-2077.

5 R. Martin, C. Cordova, J. A. San Roman, B. Gutierrez, V. Cachofeiro and M. L. Nieto, J. Mol. Cell. Cardiol., 2014, 72, 250-262.

6 T. Tian, X. Liu, E. Lee, J. Sun, Z. Feng, L. Zhao and C. Zhao, Arch. Pharmacal Res., 2017, 40, 458-468.

7 J. L. Maia, R. C. P. Lima-Junior, C. M. Melo, J. P. David, J. M. David, A. R. Campos, F. A. Santos and V. S. N. Rao, Pharmacol. Res., 2006, 54, 282-286.

8 X. Chuan, Q. Song, J. Lin, X. Chen, H. Zhang, W. Dai, B. He, X. Wang and Q. Zhang, Mol. Pharmaceutics, 2014, 11, 36563670 .

9 J. Fang, H. Nakamura and H. Maeda, Adv. Drug Delivery Rev., 2011, 63, 136-151.
10 D. Lee, J. H. Na, S. Lee, C. M. Kang, H. N. Kim, S. J. Han, H. Kim, Y. S. Choe, K. Jung, K. C. Lee, K. Choi, I. C. Kwon, S. Y. Jeong, K. Lee and K. Kim, Mol. Pharmaceutics, 2013, 10, 2190-2198.

11 R. Ngoune, A. Peters, D. von Elverfeldt, K. Winkler and G. Puetz, J. Controlled Release, 2016, 238, 58-70.

12 C. Barrera, A. P. Herrera and C. Rinaldi, J. Colloid Interface Sci., 2009, 329, 107-113.

13 Z. Ahmad, S. Lv, Z. Tang, A. Shah and X. Chen, J. Biomater. Sci., Polym. Ed., 2016, 27, 40-54.

14 Y. Li, J. Lin, X. Yang, Y. Li, S. Wu, Y. Huang, S. Ye, L. Xie, L. Dai and Z. Hou, ACS Appl. Mater. Interfaces, 2015, 7, 17573-17581.

15 Y. Li, J. Lin, G. Liu, Y. Li, L. Song, Z. Fan, X. Zhu, G. Su and Z. Hou, RSC Adv., 2016, 6, 86717-86727.

16 X. Wang, X. Gu, H. Wang, Y. Sun, H. Wu and S. Mao, Eur. J. Pharm. Sci., 2017, 96, 255-262.

17 R. K. Thapa, H. T. Nguyen, J. Jeong, B. S. Shin, S. K. Ku, H. Choi, C. S. Yong and J. O. Kim, Nanomedicine, 2016, 13, 885-896.

18 M. J. Ernsting, W. L. Tang, N. MacCallum and S. D. Li, Bioconjugate Chem., 2011, 22, 2474-2486.

19 H. H. Y. Tong, H. B. Wu, Y. Zheng, J. Xi, A. H. L. Chow and C. K. Chan, Int. J. Pharm., 2008, 355, 195-202.

20 Y. X. Wang and Y. B. Tan, J. Drug Delivery Sci. Technol., 2016, 36, 183-191.

21 M. Lou, L. N. Zhang, P. G. Jia, F. Q. Feng, J. H. Liu, C. Yang, B. F. Li and L. Wang, Biomed. Pharmacother, 2016, 84, 1-9.

22 Y. Han, Z. He, A. Schulz, T. K. Bronich, R. Jordan, R. Luxenhofer and A. V. Kabanov, Mol. Pharmaceutics, 2012, 9, 2302-2313.

23 A. Ariza, M. I. Montanez, T. D. Fernandez, J. R. Perkins and C. Mayorga, Curr. Pharm. Des., 2016, 22, 6773-6783.

24 S. M. Atkinson and A. Nansen, Basic Clin. Pharmacol. Toxicol., 2017, 120, 108-114.

25 T. M. Allen and P. R. Cullis, Science, 2004, 303, 1818-1822.

26 J. Du, T. Sun, W. Song, J. Wu and J. Wang, Angew. Chem., Int. Ed., 2010, 49, 3621-3626.

27 M. Karimi, M. Eslami, P. Sahandi-Zangabad, F. Mirab, N. Farajisafiloo, Z. Shafaei, D. Ghosh, M. Bozorgomid, F. Dashkhaneh and M. R. Hamblin, WIREs Nanomedicine and Nanobiotechnology, 2016, 8, 696-716. 